\title{
An experimental demonstration of learning without awareness
}

\author{
L. W. BUCKALEW, S. H. FINESMITH, AND DAVID A \\ SISEMORE, DEPARTMENT OF PSYCHOLOGY, UNIVERSITY \\ OF SOUTHERN MISSISSIPPI, Hattiesburg, Miss. 39401
}

In an attempt to demonstrate the existence of learning without awareness, 18 college students, instructed that ESP was to be studied, were given four trials of guessing card suits in standard decks. Motivation and knowledge of results were afforded. Unknown to the students, decks were arranged with suit sequences. A general learning curve resulted, with no indication of awareness, and group performance significantly exceeded probability expectancy. Existence of learning without awareness was supported.

Hilgard \& Bower (1966) indicated that the phenomena included in the spread of effect came nearest to a discovery in the whole of Thorndike's work. One experimental interest stimulated by introduction of this discovery was learning without awareness. Based on evidence that a satisfying aftereffect could strengthen the connection which it followed and to which it belonged in cases where the learner did not know what the connection was, Thorndike \& Rock (1934) carried out several experiments which purported to show that learning could proceed without awareness of what was being learned or intent to learn. Miller (1940), in investigating learning without awareness, found that acceleration of a learning curve was affected by changes in motivation, and relative strengths of certain motivations for such learning were generally comparable to those for learning with awareness.

Adams (1957) presented a review and classification of the psychological processes to which behavior without awareness has been applied. It was concluded that many kinds of such behavior do occur, and the establishment of conditions under which certain types can be demonstrated and measured remains an "interesting and important challenge to experimental ingenuity." A renewal of interest in learning without awareness was concluded by Postman \& Sassenrath (1961), who stated that adherence to the assumption that modification of behavior must be preceded by a correct understanding of the environmental contingencies was outmoded and unnecessary.

The present experiment attempted to demonstrate the existence of learning without awareness, operationally defined as performance, when plotted on a graph, that suggested a learning curve in which the final trial (s) was significantly better than probability expectancy, the reason for which could not be verbalized by Ss. The hypothesis for this experiment was that learning without awareness does occur in response to a card suit-guessing task which incorporated sequenced decks.

Subjects. Eighteen college students, enrolled in an experimental psychology course, served as Ss. Pairing of students resulted in nine pairs, with each student alternating as $\mathbf{S}$ and $\mathbf{E}$.

Materials. Nine decks of standard playing cards were used.

Procedure. The entire experiment, introduced as an investigation into ESP, was completed in approximately $2 \mathrm{~h}$. A brief introduction to the area of extra-sensory perception was given, and the students were told to test each other's ability to correctly guess the suit of each card in a standard playing deck. Each student served as $E$ for one practice trial and as $\mathbf{S}$ for one trial. All trials were run under a telepathy condition, in which $E$ looked at each card

Table 1

Average Number of Correct Guesses per Trial Compared with Probability Expectancy

\begin{tabular}{ccccc} 
Trial & Expected & Observed & df & t \\
\hline 1 & 13.00 & 13.22 & 17 & .29 \\
2 & 13.00 & 13.33 & 17 & .44 \\
3 & 13.00 & 14.67 & 17 & $2.21^{*}$ \\
4 & 13.00 & 14.69 & 15 & $2.09^{*}$ \\
\hline
\end{tabular}

${ }^{*} p<.05$

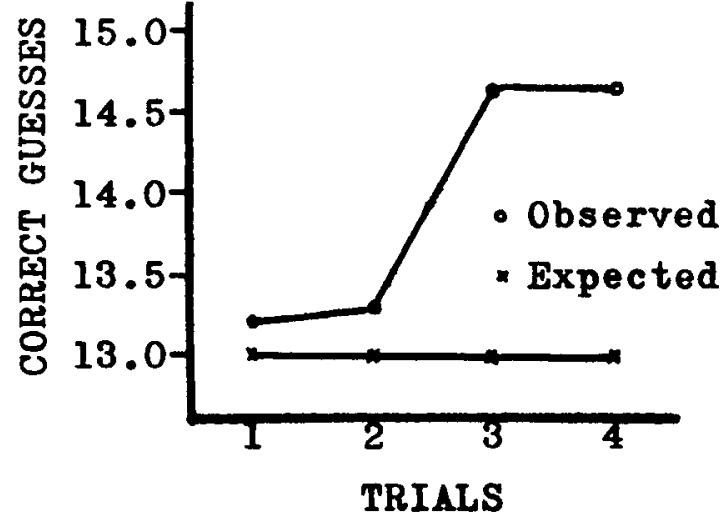

Fig. 1. Average group performance in guessing card suits on successive trials, as compared to probability expectancy.

before $S$ guessed and attempted to mentally transmit the card suit to $S$. Careful records were obtained of the correct guesses of each $S$, and data sheets were collected following each trial.

After practice trials were completed, a general discussion of the existence of ESP was stimulated among the students. Rhine's criteria for BSP were stated, and data from the practice trials were examined in this framework and that of the normal probability curve. Students were then asked to be really fair about the issue and give ESP a real chance to manifest itself under favorable conditions. Motivation (to show latent ESP ability) was introduced by promising an " $\mathrm{A}$ " on the final exam to any student who performed at or beyond the .01 level.

Students were then instructed to run four telepathy condition trials as $S$ and four as $E$, with members of each pair alternating as $S$ and $E$. After each trial, data sheets were collected and a fresh deck introduced. The essential element of the procedure was that, unknown to the students, each deck was a prearranged sandwich deck, containing eight sequences of three cards each (heart, spade, diamond) sandwiched in with the rest of the deck randomly arranged around them. Knowledge of results was afforded, with all Es directed to respond "right" or "wrong" following each guess by the S. Following completion of all trials, each pair of students was interrogated concerning their insights into factors thought to affect performance.

Results. Individual data sheets supplied the number of correct guesses for each trial. A trial was completed when all students had served in the capacity of $S$. Since each student served as $S$ four times, there were four trials, each with a total of $18 \mathrm{Ss}$, except for the last trial on which there was one less pair. Table 1 indicates the average group performance on each of the four trials. Practice trials were not included, as they were run only to introduce the nature of the experiment and did not involve the sequenced decks used on all other trials.

Since there were four suits of cards in a standard deck, and only the suit was guessed, normal probability dictated that approximately 13 of the 52 responses per deck would be correct. If probability was the main factor in operation, the group average number of correct responses should closely approximate 13.0 for all trials. The $t$ test was used to compare each trial mean with probability expectancy, and a one-tailed test indicated that Trial 3 and 4 means were significantly greater than the dictates of probability. Figure 1 reveals group data for each trial as compared to the predictions of normal probability. Within the group data, Ss ranged from an average of 11.25 to 19.25 correct guesses for the four trials.

Discussion. The general shape of the curve, as revealed in Fig. 1, suggested a definite learning function for the group of students. However, limitations of (1) the number of Ss, and (2) the number of trials reduced the clarity of this function. With the exception of the difference between Trials 2 and 3 , a gradual rise in level of proficiency was indicated. It was suggested that the relatively large change in the slope of the curve at this point represented the period in which a majority of Ss actually learned, without awareness of it, the 
general nature of the sequenced decks. It was inferred that a correct guess of the suit of a card served as a cuc to the suit of the following card. In short, Ss learned relationships within series of cards, though without awareness of the process.

The performance of Ss, as evidenced in Table 1, was significantly higher (beyond .05) than that dictated by normal probability. Comparison of probability expectancy and observed group performance indicated an achieved level of guessing for Trials 3 and 4 that could not be accounted for by chance. By design, this significant performance, facilitated through motivation and immediate knowledge of results, was suggested to be a manifestation of learning.

Postexperiment interrogation of each pair of Ss revealed that no student was able to verbalize knowledge of the sequenced decks. In fact, no student contributed any information concerning factors thought to affect performance, outside of an ESP framework. Several students who performed at a high level were very reluctant to discard the personal hypothesis that they had some definite amount of ESP. With the information obtained through interrogation, it was concluded that the learning which took place was without verification of awareness. However, the use of verbal interrogation and report as a criterion for awareness was, perhaps, a vulnerable point in procedure. Miller (1940) pointed out that this criterion was adequate, but it entailed the difficulties and dangers of introspective report.
It was concluded that learning did occur, as evidenced in a significant level of performance when compared to probability expectancy, and that this learning was without verification of awareness. Suggestions of improvement for further studies in this area include use of (1) a larger number of Ss, (2) a greater number of trials (10 or more), and (3) a less vulnerable index of awareness.

\section{REFERENCES}

ADAMS, J. K. Laboratory studies of behavior without awareness. Psychol Bulh, 1957, 54, 383-405.

HILGARD, E. R., \& BOWER, G. H. Theories of learning. (3rd ed.) New York: Appleton-Century-Crofts, 1966.

MILLER, J. G. The role of motivation in learning without awareness. Amer. J. Psychol, 1940, 53, 229-239.

POSTMAN, L., \& SASSENRATH, J. The automatic action of verbal rewards and punishments. J. gen. Psychol, 1961, 65, 109-136.

THORNDIKE, E. L., \& ROCK, R. T. Learning without awareness of what is being learned or intent to learn it. J. exp. PsychoL, 1934, 17, 1-19.

$$
\text { NOTE }
$$

1. This research was stimulated by an earlier unpublished study by $\mathbf{S}$. $\mathbf{H}$. Finesmith at Rutgers University in $\mathbf{1 9 6 2 .}$ 